\title{
Genome-wide screen for modifiers of Parkinson's disease genes in Drosophila
}

Caroline Fernandes ${ }^{1,2}$ and Yong Rao ${ }^{2^{*}}$

\begin{abstract}
Background: Mutations in parkin and PTEN-induced kinase 1 (Pink1) lead to autosomal recessive forms of Parkinson's disease (PD). parkin and Pink1 encode a ubiquitin-protein ligase and a mitochondrially localized serine/ threonine kinase, respectively. Recent studies have implicated Parkin and Pink1 in a common and evolutionarily conserved pathway for protecting mitochondrial integrity.

Results: To systematically identify novel components of the PD pathways, we generated a genetic background that allowed us to perform a genome-wide F1 screen for modifiers of Drosophila parkin (park) and Pink1 mutant phenotype. From screening $\sim 80 \%$ of the fly genome, we identified a number of cytological regions that interact with park and/or Pink1. Among them, four cytological regions were selected for identifying corresponding PDinteracting genes. By analyzing smaller deficiency chromosomes, available transgenic RNAi lines, and P-element insertions, we identified five PD-interacting genes. Among them, opa 1 and $d r p 1$ have been previously implicated in the PD pathways, whereas debra (dbr), Pi3K21B and $\beta 4$ GalNAcTA are novel PD-interacting genes.

Conclusions: We took an unbiased genetic approach to systematically isolate modifiers of PD genes in Drosophila. Further study of novel PD-interacting genes will shed new light on the function of PD genes and help in the development of new therapeutic strategies for treating Parkinson's disease.
\end{abstract}

\section{Background}

Parkinson's disease (PD) is the second most common neurodegenerative disease. It is characterized by the loss of nigral dopaminergic neurons. Mutations in Pink1 and Parkin cause autosomal recessive early-onset Parkinson's disease in humans [1,2]. Together mutations in these genes account for greater than $50 \%$ of familial Parkinson disease (PD) and $\sim 20 \%$ of early-onset sporadic cases [3-5]. Recent studies on characterizing the function of Parkin and Pink1 have significantly advanced our understanding of PD pathogenesis.

Parkin has E3-ubiquitin ligase activity, and is shown to degrade abnormally folded proteins [6]. For instance, Parkin ubiquitinates and degrades proteins such as CDCrel-1 [7], Parkin-associated endothelin receptor-like (Pael) receptor [8], $\alpha$-synuclein [9], synphilin-1 [10], and cyclin E [11]. Thus, Parkin dysfunction in regulating the

\footnotetext{
* Correspondence: yong.rao@mcgill.ca

${ }^{2}$ McGill Centre for Research in Neuroscience, Department of Neurology and Neurosurgery, Department of Medicine, McGill University Health Centre, 1650 Cedar Avenue, Montreal, Quebec H3G 1A4, Canada

Full list of author information is available at the end of the article
}

level of other proteins or itself through protein degradation may contribute to PD pathogenesis.

Pink1 is a mitochondria-localized serine/threonine kinase $[2,12,13]$. A recent study suggests that Pink1 directly phosphorylates Parkin [14]. In addition, Pink1 may directly or indirectly induce the phosphorylation of the HSP75 chaperone TRAP1 [12] and the mitochondrial protease HtrA2 [13].

Accumulated evidence supports that Pink1 and Parkin act together in a common and conserved pathway to protect mitochondrial integrity (for review, see [15]). For instance, it is reported that overexpression of Drosophila Parkin (park) could rescue mitochondrial defects caused by Pink1 mutations both in Drosophila [16-19] and mammalian systems $[20,21]$. Recent studies also indicate that Pink1-dependent recruitment of Park into mitochondria is required for the clearance of damaged mitochondria [22-25].

Drosophila melanogaster has proven to be a powerful model system for understanding the function of PD genes. Several PD genes such as park, Pink1, LRRK2 and HtrA1 have orthologs in Drosophila. Interfering with

\section{() Biomed Central}


their function caused PD-like phenotypes in Drosophila [17-19,26-29]. Genetic studies in Drosophila have begun to reveal new targets for the development of new therapeutic approaches to treat PD. For instance, Pallanck and colleagues previously conducted a genetic screen to isolate modifiers of partial lethality caused by complete loss of park in Drosophila [30]. From 1400 P-element insertions affecting less than $10 \%$ of the fly genome, they identified several genes that regulate oxidative stress and innate immune responses [30].

In this study, we conducted a systematic genetic screen to isolate park- and/or Pink1-interacting regions that cover $\sim 80 \%$ of the entire fly genome. We generated a genetic background in which park or Pink1 was knocked down. The availability of this genetic tool allowed us to perform a F1 genetic screen to identify cytological regions on the $2^{\text {nd }}$ and $3^{\text {rd }}$ chromosome that interact with park and/or Pink1. We found that 31 cytological regions modify both park and Pink1 wing-posture phenotype. In addition, 21 cytological regions showed interactions with both Pink1 and park in adult lethality test. We then selected four cytological regions for fine mapping, which identified two known PD-interacting genes opa 1 and $d r p 1$, and three novel PD-interacting genes debra, Pi3K21B and $\beta 4$ GalNAcTA.

\section{Methods}

\section{Drosophila stocks}

UAS-Pink1-RNAi, UAS-park RNAi and other transgenic RNAi lines were obtained from the VDRC stock center. A collection of deficiencies uncovering $>92 \%$ of cytological regions on $2^{\text {nd }}$ and $3^{\text {rd }}$ chromosomes were obtained from the Bloomington Drosophila Stock Center. Smaller deficiencies and P-element insertions mapped within large PD-interacting cytological regions were also obtained from the Bloomington Drosophila Stock Center. Balancer stocks CyO,GAL80 and TM3, GAL80 were provided by D.van Meyel. The park $^{\text {edpk } \Delta 21} / T M 3, S b$ line was provided by $M$. Guo (UCLA). Pink1 $1^{\text {B9 }} /$ FM7,Act-GFP and park ${ }^{25} /$ TM3,Sb stocks were provided by T. Fon. $D f(2 R) \beta 4 G a l N A c T A^{[20.1]}$ and $\beta 4$ GalNAcTA $A^{4.1}$ lines were obtained from N. Haines.

\section{Genetics}

To knock down Pink1 or park, tubulin-GAL4 (tubGAL4) flies were crossed with UAS-Pink1-RNAi or UASpark-RNAi flies to ubiquitously express Pink1-RNAi or park RNAi. Since fly stocks with ubiquitous expression of Pink1-RNAi or park RNAi under control of tub-GAL4 are not healthy, genetic crosses were performed to generate UAS-Pink1-RNAi/CyO,GAL80; tub-GAL4/TM3,Sb and UAS-park-RNAi;tub-Gal4/TM3,Sb,GAL80 stocks, in which GAL4 is inhibited by GAL80 to prevent the expression of UAS-Pink1-RNAi or UAS-park-RNAi in parental stocks [31].
F1 screen was performed by crossing individual deficiency lines from $2^{\text {nd }}$ and $3^{\text {rd }}$ chromosome deficiency kits with UAS-Pink1-RNAi/CyO,GAL80; tub-GAL4/TM3, $S b$ or UAS-park-RNAi;tub-Gal4/TM3,Sb,GAL80 flies. The F1 progeny in Pink1-RNAi background were reared at $25^{\circ}$, and the $\mathrm{F} 1$ progeny in park-RNAi background were kept at $29^{\circ}$. F1 progeny were collected for 4-6 days and separated according to their date of eclosion. The modification of wing-posture phenotype by each deficiency chromosome was scored on post-eclosion day 3 for Pink 1 screen and on post-eclosion day 6 for park screen. Wing posture phenotype in both male and female F1 flies was scored, and the modifying effect on penetrance was determined by counting the percentage of both held-up-wing flies and drooped-wing flies. For park and Pink1 screen, 212 and 217 deficiencies in the deficiency kit were screened, respectively.

Selected deficiency lines were also crossed with Pink $1^{\mathrm{B} 9} /$ FM7,Act-GFP female flies. F1 progeny were scored for the modification of the wing-posture phenotype. The F1 progeny were also scored for adult lethality test.

\section{Analysis of wing phenotype, longevity and fertility}

For analysis of abnormal wing phenotype, 20 flies were placed per vial. Flies with both wings held-up or drooped were counted.

For longevity test, flies were collected upon eclosion and transferred to new vials every 4-6 days. Mortality was scored daily. The assay was performed in triplicate. Survival curves were plotted using GraphPad software.

To test fertility of male flies, individual male flies were crossed with three (w1118) virgin females. After 10 days, the number of vials with progeny were counted.

\section{Statistical Analysis}

Student's t-test was used for statistical analysis.

\section{Results}

\section{Characterization of park and Pink1 knockdown phenotypes}

Previous studies show that loss of park or loss of Pink1 caused similar phenotypes, such as abnormal wing morphology, male sterility, reduced climbing ability, decreased longevity and loss of dopaminergic neurons [17-19,29]. To generate a "park-inhibited" or "Pink1inhibited" background suitable for systematic F1 genetic screen, we used the GAL4-UAS system [32] to knock down the level of Pink1 or park in flies.

Consistent with previous reports [17-19,29], we found that ubiquitous knockdown of Pink1 or park by expressing UAS-park-RNAi or UAS-Pink1-RNAi transgenes under control of the tub-GAL4 driver, caused male sterility (compared to $100 \%$ fertility in wild-type control, 

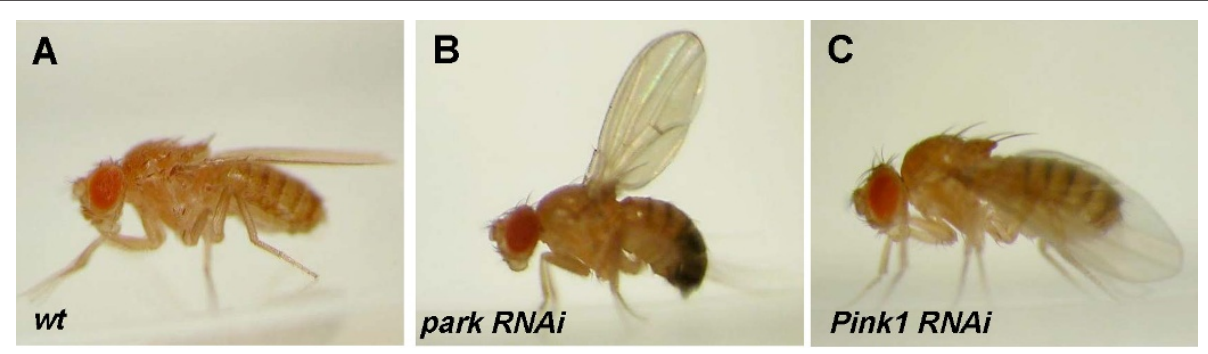

D

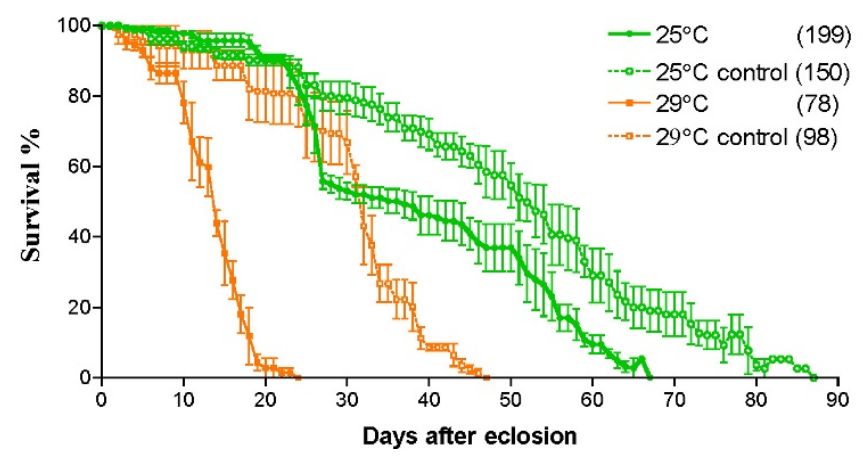

Figure 1 Knockdown of PD genes induces a wing position phenotype in adult flies. A-C, Ubiquitous knockdown of PD genes in flies induces an abnormal wing position phenotype (i.e. held up or drooped wing). A. Wild-type wing position in flies carrying only the tub-GAL4 driver. B, A park knockdown fly (i.e. a fly carrying both the tub-GAL4 driver and UAS-park-RNAi transgene). The "held-up" wing position phenotype was observed when UAS-park-RNAi was ubiquitously expressed under control of the tub-GAL4 driver. C, The "drooped" wing phenotype was observed in a Pink1 knockdown fly (i.e. a fly carrying both the tub-GAL4 driver and UAS-Pink1-RNAi transgene). D, Survival curves of park knockdown flies (solid line) and control flies (i.e. flies carrying only the UAS-park transgene) (dashed line). The experiments were performed at $25^{\circ}$ $\mathrm{C}$ (green) and $29^{\circ} \mathrm{C}$ (red). Numbers in brackets represent sample numbers. Error bars represent SEM.

Pink1 and park knockdown flies showed $14.3 \%$ and $44.4 \%$ fertility, respectively), reduced life span, and abnormal wing posture (i.e. held up or drooped) (Figure 1). Those phenotypes resembled that observed in park and Pink1 loss-of-function mutants [17-19,29].

We then tested if the penetrance and severity of above phenotypes could be enhanced by increasing the expression level of the UAS-park-RNAi transgene. This was achieved by elevating temperature, which increases the activity of GAL4 leading to higher expression of UAStransgenes [32]. Indeed, we found that increasing the expression level of park-RNAi significantly enhanced the phenotype. The penetrance of wing-posture phenotype in park knockdown flies was increased from $\sim 2.1 \%$ at $25^{\circ} \mathrm{C}$ to $\sim 22.4 \%$ at $29^{\circ} \mathrm{C}$. The maximal life span of park knockdown flies was further reduced from $\sim 67$ days at $25^{\circ} \mathrm{C}$ to $\sim 17$ days at $29^{\circ} \mathrm{C}$. The fertility of male park knockdown flies was also reduced from $\sim 44.4 \%$ at $25^{\circ} \mathrm{C}$ to $\sim 30 \%$ at $29^{\circ} \mathrm{C}$.

We also examined the effect of increasing the level of Pink1-RNAi transgene on wing posture, male sterility and longevity. In Pink1 knockdown flies, the penetrance of wing-posture phenotype was increased from $\sim 2.9 \%$ at room temperature to $\sim 91 \%$ at $29^{\circ} \mathrm{C}$. The maximal life span of Pink1 knockdown flies was reduced from $\sim 55$ days at room temperature to $\sim 18$ days at $29^{\circ} \mathrm{C}$. The fertility of male Pink1 knockdown flies was also decreased from $\sim 14.3 \%$ at room temperature to $0 \%$ at $29^{\circ} \mathrm{C}$.

\section{F1 screen for modifiers of the park knockdown phenotype}

To identify novel modifiers of the PD pathway, we set out to conduct a systematic screen to identify cytological regions on the $2^{\text {nd }}$ and $3^{\text {rd }}$ chromosome that interact with park (Figure 2).

Prior to the screen, we examined if the park knockdown mutant background is sensitive to the reduction in the dosage of known genes in the pathway. We found that reducing the level of endogenous park substantially increased the penetrance of the park-RNAi-induced wing posture phenotype from $\sim 15 \%(\mathrm{n}=76)$ (genotype: park $R N A i ;+/+)$ to $\sim 43 \%(\mathrm{n}=97)$ (genotype: park RNAi; park $\left.{ }^{25} /+\right)(\mathrm{P}<0.05)$. Since Pink1 and park have previously been shown to act in a common pathway [17-19], we also tested if the park knockdown background is sensitive to a reduction in the level of Pink1. Indeed, we found that Pink1 heterozygostiy significantly enhanced the penetrance of the park-RNAi-induced 


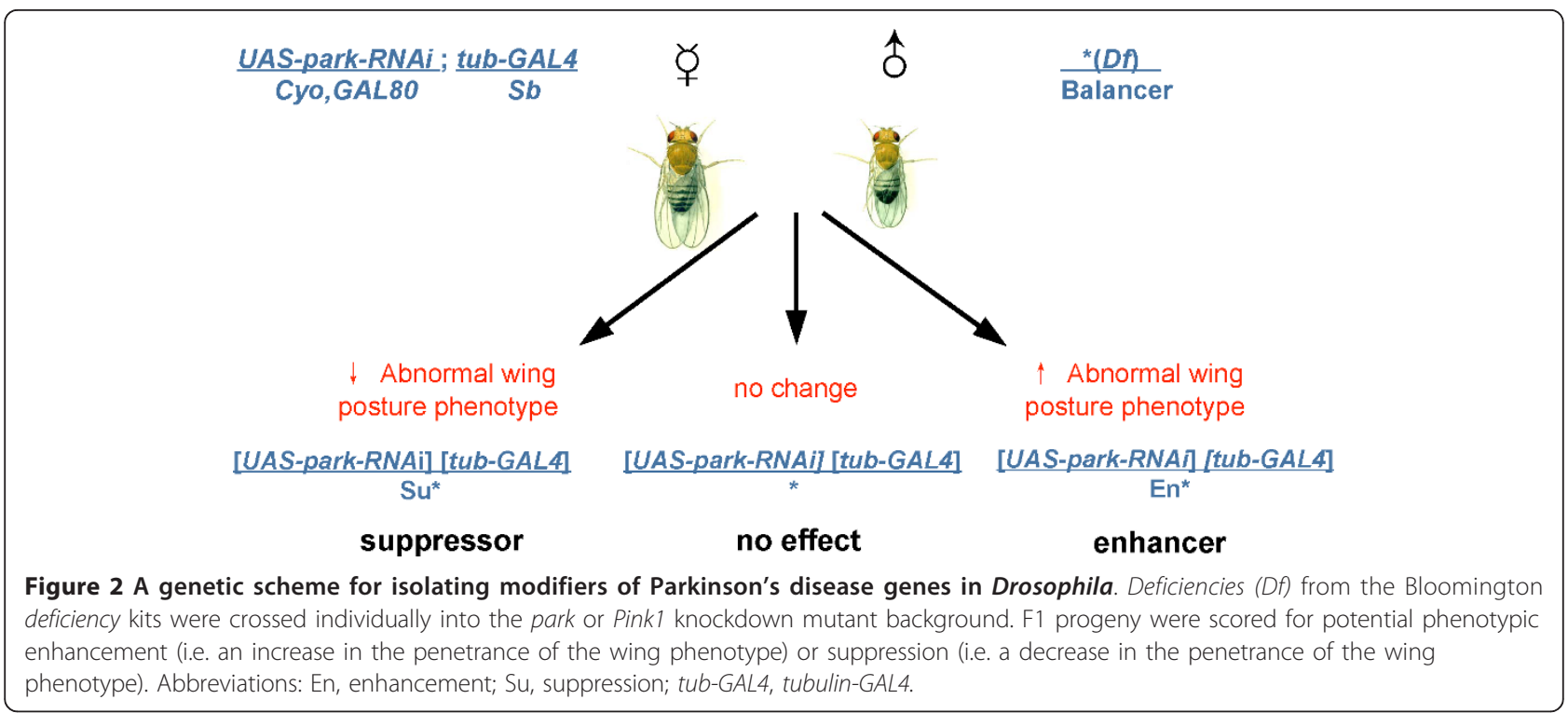

wing posture phenotype from $\sim 13 \%(\mathrm{n}=90)$ (genotype: +/+; park RNAi) to $\sim 40 \%(\mathrm{n}=32)$ (genotype: $\operatorname{Pink} 1^{B 9} /+$; park $R N A i)(\mathrm{P}<0.01)$.

To systematically identify modifiers of this park wingposture phenotype, we crossed a large collection of deficiencies on the $2^{\text {nd }}$ and $3^{\text {rd }}$ chromosome into the park knockdown mutant background. In each deficiency chromosome, a portion of cytological regions was deleted. Thus, crossing a deficiency chromosome into the park knockdown background led to $50 \%$ reduction in the dosage of genes located within the deleted cytological region.

From this screen, we identified 26 cytological regions that enhanced the park wing-posture phenotype (Table 1 ), and 53 cytological regions that suppressed the wingposture phenotype (Table 2). We also found that reducing the dosage of genes by $50 \%$ in 48 cytological regions in park knockdown flies caused lethality prior to the adult stage (Table 3 ). No such adult lethality was observed when park was knocked down alone, or the dosage of those 48 cytological regions was reduced by $50 \%$ in wild type background.

\section{F1 screen for modifiers of the Pink1 knockdown phenotype}

Above deficiencies were also screened using the Pink1 knockdown mutant background. Pink1 knockdown mutant flies displayed the wing-posture phenotype at the penetrance of $\sim 64 \%(\mathrm{n}=314)$ at $25^{\circ} \mathrm{C}$. Among 26 enhancer-containing cytological regions identified from the Park screen (Table 1), 8 cytological regions, when reduced by $50 \%$ in dosage, also enhanced the penetrance of the Pink 1 knockdown wing phenotype (Table 4). This
Table 1 Enhancers of the park-RNAi wing phenotype

\begin{tabular}{|c|c|c|}
\hline Deficiencies & Breakpoints & Strength of modification ${ }^{a}$ \\
\hline Df(2L)net-PMF & $21 \mathrm{~A} 1 ; 21 \mathrm{~B} 7-8$ & ++ \\
\hline$D f(2 L) B S C 28$ & 23C5-D1;23E2 & ++++ \\
\hline$D f(2 L) c l-h 3$ & 25D2-4;26B2-5 & ++ \\
\hline$D f(2 L) B S C 7$ & 26D10-E1;27C1 & ++ \\
\hline$D f(2 L) E D 611$ & 29B4;29C3 & ++ \\
\hline$D f(2 L) B S C 17$ & $30 C 3-5 ; 30 F 1$ & ++ \\
\hline$D f(2 L) M d h$ & 30D-30F;31F & +++++ \\
\hline$D f(2 L) B S C 50$ & 30F5;31B1 & ++++++ \\
\hline$D f(2 L) F C K-20$ & $32 \mathrm{D} 1 ; 32 \mathrm{~F} 1-3$ & +++ \\
\hline Df(2R)nap9 & $42 \mathrm{~A} 1-2 ; 42 \mathrm{E} 6-\mathrm{F} 1$ & +++++ \\
\hline$D f(2 R) c n 9$ & $42 \mathrm{E} ; 44 \mathrm{C}$ & ++ \\
\hline$D f(2 R) H 3 E 1$ & $44 \mathrm{D} 1-4 ; 44 \mathrm{~F} 12$ & +++ \\
\hline Df(2R)en30 & $48 \mathrm{~A} 3-4 ; 48 C 6-8$ & +++ \\
\hline Df(2R)BSC39 & 48C5-D1;48D5-E1 & ++++ \\
\hline Df(2R)BSC161 & $54 \mathrm{~B} 2 ; 54 \mathrm{~B} 17$ & ++++ \\
\hline Df(2R)Exel7162 & $56 F 11 ; 56 F 16$ & ++++ \\
\hline$D f(2 R) 59 A D$ & 59A1-3;59D1-4 & +++ \\
\hline$D f(3 L) A C 1$ & 67A2;67D11-13 & +++++ \\
\hline Df(3L)XS533 & $76 \mathrm{~B} 4 ; 77 \mathrm{~B}$ & ++ \\
\hline Df(3L)BSC249 & 79B2;79D2 & ++ \\
\hline Df(3R)BSC47 & 83B7-C1;83C6-D1 & ++ \\
\hline Df(3R)Tp/10 & $83 \mathrm{C} 1-2 ; 84 \mathrm{~B} 1-2$ & ++ \\
\hline$D f(3 R) B S C 43$ & 92F7-93A1;93B3-6 & ++ \\
\hline Df(3R)BSC56 & 94E1-2;94F1-2 & ++ \\
\hline Df(3R)BSC137 & $95 \mathrm{~A} 2-4 ; 95 \mathrm{~A} 8-\mathrm{B} 1$ & +++ \\
\hline$D f(3 R) B S C 42$ & 98B1-2;98B3-5 & ++ \\
\hline
\end{tabular}

Each deficiency was crossed into the park RNAi background and the wing posture phenotype was scored. Crosses were maintained at $29^{\circ} \mathrm{C}$.

${ }^{a}$ Each ' + ' represents 1.0 SD from the mean pentrance (i.e. 22.4\%) observed for park RNAi alone flies. 
Table 2 Suppressors of the park-RNAi wing phenotype

\begin{tabular}{|c|c|c|}
\hline Deficiencies & Breakpoints & Strength of modification ${ }^{a}$ \\
\hline Df(2L)BSC106 & $21 \mathrm{~B} 7 ; 21 \mathrm{C} 2$ & ++++ \\
\hline$D f(2 L) d p-79 b$ & 22A2-3;22D5-E1 & ++++ \\
\hline Df(2L)ed1 & 24A2;24D4 & ++++ \\
\hline$D f(2 L) s c 19-8$ & $24 C 2-8 ; 25 C 8-9$ & ++ \\
\hline$D f(2 L) B S C 110$ & $25 \mathrm{C} 1 ; 25 \mathrm{C} 4$ & ++ \\
\hline Df(2L)BSC109 & $25 C 4 ; 25 C 8$ & ++++ \\
\hline$D f(2 L) E 110$ & 25F3-26A1;26D3-11 & ++ \\
\hline$D f(2 L) B S C 142$ & $28 \mathrm{C} 3 ; 28 \mathrm{D} 3$ & ++++ \\
\hline$D f(2 L) B S C 143$ & 31B1;31D9 & ++ \\
\hline$D f(2 L) B S C 145$ & $32 \mathrm{C} 1 ; 32 \mathrm{C} 1$ & +++ \\
\hline$D f(2 L) b 87 e 25$ & 34B12-C1;35B10-C1 & ++++ \\
\hline $\operatorname{Df}(2 L) C^{\prime}$ & h35;h38L & ++ \\
\hline$D f(2 R) B S C 3$ & 48E12-F4;49A11-B6 & ++++ \\
\hline Df(2R)Exel7131 & $50 \mathrm{E} 4 ; 50 \mathrm{~F} 6$ & ++++ \\
\hline$D f(2 R) B S C 550$ & $53 \mathrm{C} 1 ; 53 \mathrm{C} 6$ & ++ \\
\hline$D f(2 R)$ robl-c & $54 \mathrm{~B} 17-\mathrm{C} 4 ; 54 \mathrm{C} 1-4$ & ++ \\
\hline$D f(2 R) B S C 45$ & 54C8-D1;54E2-7 & ++++ \\
\hline$D f(2 R) P 34$ & $55 \mathrm{E} 2-4 ; 56 C 1-11$ & ++++ \\
\hline$D f(2 R) A A 21$ & 56F9-17;57D11-12 & ++ \\
\hline$D f(2 R) B S C 155$ & $60 \mathrm{~B} 9 ; 60 \mathrm{C} 4$ & +++ \\
\hline$D f(2 R) M 60 E$ & 60E2-3;60E11-12 & ++ \\
\hline Df(3L)Aprt-1 & $62 \mathrm{~A} 10-\mathrm{B} 1 ; 62 \mathrm{D} 2-5$ & ++++ \\
\hline$D f(3 L) B S C 181$ & $62 \mathrm{~A} 11 ; 62 \mathrm{~B} 7$ & ++++ \\
\hline$D f(3 L) X D / 98$ & 65A2;65E1 & ++ \\
\hline$D f(3 L) B S C 33$ & $65 \mathrm{E} 10-\mathrm{F} 1 ; 65 \mathrm{~F} 2-6$ & ++ \\
\hline$D f(3 L) p b l-X_{1}$ & $65 F 3 ; 66 \mathrm{~B} 10$ & ++++ \\
\hline$D f(3 L) 66 C-G 28$ & $66 \mathrm{~B} 8-9 ; 66 C 9-10$ & +++ \\
\hline$D f(3 L) h-i 22$ & 66D10-11;66E1-2 & +++ \\
\hline$D f(3 L) S C f-R 6$ & $66 \mathrm{E} 1-6 ; 66 \mathrm{~F} 1-6$ & ++ \\
\hline$D f(3 L) B S C 283$ & $67 C 7 ; 67 D 5$ & +++ \\
\hline$D f(3 L)$ eyg $[C 1]$ & 69A4-5;69D4-6 & ++++ \\
\hline$D f(3 L) B S C 10$ & 69D4-5;69F5-7 & ++++ \\
\hline$D f(3 L) B S C 12$ & 69F6-70A1;70A1-2 & ++ \\
\hline$D f(3 L) f z-G F 3 b$ & $70 C 1-2 ; 70 D 4-5$ & +++ \\
\hline$D f(3 L) X G 5$ & $71 C 2-3 ; 72 B 1-C 1$ & ++ \\
\hline$D f(3 L) f z 2$ & 75F10-11;76A1-5 & ++++ \\
\hline Df(3L)ME107 & 77F3;78C8-9 & +++ \\
\hline Df(3R)ME15 & $81 F 3-6 ; 82 F 5-7$ & ++ \\
\hline$D f(3 R) 3-4$ & $82 F 3-4 ; 82 F 10-11$ & ++++ \\
\hline$D f(3 R) p-X T 103$ & $85 A 2 ; 85 C 1-2$ & ++++ \\
\hline$D f(3 R) B S C 38$ & $85 F 1-2 ; 86 C 7-8$ & ++ \\
\hline Df(3R)sbd105 & 88F9-89A1;89B9-10 & ++ \\
\hline Df(3R)sbd104 & $89 B 5 ; 89 C 2-7$ & ++ \\
\hline$D f(3 R) P 115$ & 89B7-8;89E7 & +++ \\
\hline Df(3R)Exel9012 & 94E9;94E13 & +++ \\
\hline Df(3R)Exel6195 & $95 \mathrm{~A} 4 ; 95 \mathrm{~B} 1$ & ++ \\
\hline Df(3R)Exel9014 & $95 \mathrm{~B} 1 ; 95 \mathrm{D} 1$ & +++ \\
\hline Df(3R)Exel6196 & $95 \mathrm{C} 12 ; 95 \mathrm{D} 8$ & +++ \\
\hline Df(3R)crb-F89-4 & 95D7-D11;95F15 & +++ \\
\hline$D f(3 R) s / 08$ & $96 \mathrm{~A} 2-7 ; 96 \mathrm{D} 2-4$ & ++++ \\
\hline Df(3R)Exel6202 & 96C9;96Е2 & +++ \\
\hline Df(3R)Exel6203 & 96E2;96E6 & ++++ \\
\hline$D f(3 R) B 81$ & 99D3;3Rt & ++++ \\
\hline
\end{tabular}

${ }^{a}$ Each ' + ' represents 0.5 SD from the mean ( 22.4\%) observed for park RNAi alone flies.
Table 3 List of deficiencies showing lethal interactions with park knockdown

\begin{tabular}{|c|c|}
\hline Deficiencies & Breakpoints \\
\hline$D f(2 L) B S C 16$ & 21C3-4;21C6-8 \\
\hline Df(2L)ast2 & 21D1-2;22B2-3 \\
\hline$D f(2 L) B S C 37$ & 22D2-3;22F1-2 \\
\hline$D f(2 L) d p p[d 14]$ & 22E4-F2;22F3-23A1 \\
\hline$D f(2 L) C 144$ & $22 \mathrm{~F} 4-23 \mathrm{~A} 1 ; 23 \mathrm{C} 2-4$ \\
\hline Df(2L)Exel6011 & 25C8;25D5 \\
\hline$D f(2 L) N 22-14$ & $29 C 1-2 ; 30 C 8-9$ \\
\hline$D f(2 L) J 2$ & $31 \mathrm{~B} ; 32 \mathrm{~A}$ \\
\hline Df(2L)cact-255rv64 & $35 F-36 A ; 36 D$ \\
\hline Df(2L)TW137 & 36C2-4;37B9-C1 \\
\hline $\ln (2 R) b w[V D e 2 L] C y[R]$ & h42-h43;42A2-3 \\
\hline$D f(2 R) N p 5$ & 44F10;45D9-E1 \\
\hline$D f(2 R) B S C 29$ & 45D3-4;45F2-6 \\
\hline $\operatorname{Df}(2 R) \times 1$ & 46C;47A1 \\
\hline Df(2R)en-A & 47D3;48B2 \\
\hline$D f(2 R) v g-C$ & 49A4-13;49E7-F1 \\
\hline$D f(2 R) C X 1$ & $49 C 1-4 ; 50 C 23-D 2$ \\
\hline$D f(2 R) B S C 18$ & $50 \mathrm{D} 1 ; 50 \mathrm{D} 2-7$ \\
\hline Df(2R)Exel7130 & $50 \mathrm{D} 4 ; 50 \mathrm{E} 4$ \\
\hline$D f(2 R) J p 1$ & 51D3-8;52F5-9 \\
\hline$D f(2 R) B S C 49$ & 53D9-E1;54B5-10 \\
\hline$D f(2 R) P C 4$ & $55 \mathrm{~A} ; 55 \mathrm{~F}$ \\
\hline$D f(2 R)$ Egfr5 & 57D2-8;58D1 \\
\hline$D f(2 R) E D 4065$ & $60 C 8 ; 60 \mathrm{E} 8$ \\
\hline$D f(2 R) K r 10$ & $60 F 1 ; 60 F 5$ \\
\hline$D f(3 L) M 21$ & $62 \mathrm{~F} ; 63 \mathrm{D}$ \\
\hline$D f(3 L) H R 119$ & $63 \mathrm{C} 2 ; 63 \mathrm{FF}$ \\
\hline Df(3L)GN34 & 63Е6-9;64A8-9 \\
\hline$D f(3 L) Z N 47$ & $64 C ; 65 C$ \\
\hline$D f(3 L) \operatorname{vin} 5$ & $68 \mathrm{~A} 2-3 ; 69 \mathrm{~A} 1-3$ \\
\hline$D f(3 L) s t-f 13$ & 72C1-D1;73A3-4 \\
\hline$D f(3 L) 81 k 19$ & $73 \mathrm{~A} 3 ; 74 \mathrm{~F}$ \\
\hline$D f(3 L) W 10$ & 75A6-7;75C1-2 \\
\hline Df(3L)ED4978 & 78D5;79A2 \\
\hline$D f(3 L) B S C 223$ & 79A3;79B3 \\
\hline Df(3R)Exel6144 & 83A6;83B6 \\
\hline Df(3R)WIN11 & 83E1-2;84A5 \\
\hline$D f(3 R) p 712$ & 84D4-6;85B6 \\
\hline$D f(3 R) M-K x 1$ & $86 \mathrm{C} 1 ; 87 \mathrm{~B} 1-5$ \\
\hline$D f(3 R) T-32$ & $86 \mathrm{E} 2-4 ; 87 \mathrm{C} 6-7$ \\
\hline Df(3R)ry615 & 87B11-13;87E8-11 \\
\hline Df(3R)ea & 88E7-13;89A1 \\
\hline$D f(3 R) D G 2$ & 89E1-F4;91B1-B2 \\
\hline$D f(3 R) D I-B \times 12$ & 91F1-2;92D3-6 \\
\hline$D f(3 R) e-R 1$ & 93B6-7;93D2 \\
\hline Df(3R)Exel6197 & 95D8;95E5 \\
\hline Df(3R)Esp/3 & $96 F 1 ; 97 B 1$ \\
\hline$D f(3 R) / R 16$ & 97F1-2;98A \\
\hline$D f(2 L) B S C 16$ & 21C3-4;21C6-8 \\
\hline Df(2L)ast2 & 21D1-2;22B2-3 \\
\hline$D f(2 L) B S C 37$ & 22D2-3;22F1-2 \\
\hline
\end{tabular}

Above deficiencies, when crossed into the park knockdown background, significantly reduced the viability of park knockdown flies (less than five flies eclosed). 
screen also identified 9 enhancer-containing cytological regions that were not uncovered from the park screen (Table 4). Among 53 suppressor-containing cytological regions identified from the park screen (Table 2), we found that 23 cytological regions also contained suppressors of the Pink1 wing-posture phenotype (Table 5). In addition, we found that 30 cytological regions, when reduced by $50 \%$ in dosage, suppressed the Pink 1 wing phenotype but not the park wing phenotype (Table 5).

Among 50 cytological regions that caused adult lethality when their dosage was reduced by $50 \%$ in park knockdown background (Table 3), 21 cytological regions also displayed a similar lethal interaction with Pink1 knockdown (Table 6). Five cytological regions only showed lethal interactions with Pink1 but not park (Table 6).

\section{Analysis of genetic interactions using a Pink1 null mutant} allele

Cytological regions identified from above $R N A i$-based screen may contain genes that function in the Pink1/ park pathway, or genes that function in a parallel pathway that act together with the Pink1/park pathway to regulate mitochondrial function. To further characterize these cytological regions, we performed genetic analysis using a Pink1 null mutant allele to test the potential interactions between Pink1 and cytological regions that interact with both Pink1 and park in the above RNAibased screen. Among six enhancer-containing cytological regions examined, five cytological regions, when

Table 4 Enhancers of the Pink1-RNAi wing phenotype

\begin{tabular}{|c|c|c|}
\hline Deficiencies & Breakpoints & Strength of modification ${ }^{a}$ \\
\hline$D f(2 L) n e t-P M F$ & $21 \mathrm{~A} 1 ; 21 \mathrm{~B} 7-8$ & ++ \\
\hline$D f(2 L) B S C 4$ & $21 B 7-C 1 ; 21 C 2-3$ & ++ \\
\hline$D f(2 L) B S C 16$ & $21 C 3-4 ; 21 C 6-8$ & ++ \\
\hline$D f(2 L) B S C 17$ & $30 \mathrm{C} 3-5 ; 30 \mathrm{~F} 1$ & ++ \\
\hline$D f(2 L) B S C 50$ & $30 \mathrm{~F} 5 ; 31 \mathrm{~B} 1$ & +++ \\
\hline$D f(2 R) n a p 9$ & $42 \mathrm{~A} 1-2 ; 42 \mathrm{E} 6-\mathrm{F} 1$ & ++ \\
\hline$D f(2 R) c n 9$ & $42 \mathrm{E} ; 44 \mathrm{C}$ & ++ \\
\hline$D f(2 R) B S C 29$ & $45 \mathrm{D} 3-4 ; 45 F 2-6$ & ++ \\
\hline$D f(2 R) B S C 39$ & 48C5-D1;48D5-E1 & ++ \\
\hline$D f(2 R) B S C 3$ & 48E12-F4;49A11-B6 & +++ \\
\hline$D f(2 R) B S C 22$ & 56D7-E3;56F9-12 & ++ \\
\hline$D f(3 L) B S C 27$ & 65D4-5;65E4-6 & ++ \\
\hline$D f(3 L) B S C 14$ & 67E3-7;68A2-6 & +++ \\
\hline$D f(3 L) X G 5$ & $71 \mathrm{C} 2-3 ; 72 \mathrm{~B} 1-\mathrm{C} 1$ & +++ \\
\hline$D f(3 L) E D 4782$ & $75 F 2 ; 76 \mathrm{~A} 1$ & ++ \\
\hline$D f(3 L) H D 1$ & 79D3-E1;79F3-6 & ++ \\
\hline$D f(3 R) B S C 47$ & 83B7-C1;83C6-D1 & ++ \\
\hline$D f(3 R) T p / 10$ & $83 C 1-2 ; 84 B 1-2$ & ++ \\
\hline
\end{tabular}

Table 5 Suppressors of the Pink1-RNAi wing phenotype

\begin{tabular}{|c|c|c|}
\hline Deficiencies & Breakpoints & Strength of modification ${ }^{a}$ \\
\hline$D f(2 L) B S C 106$ & $21 B 7 ; 21 C 2$ & +++ \\
\hline$D f(2 L) d p-79 b$ & 22A2-3;22D5-E1 & +++++ \\
\hline$D f(2 L) J S 17$ & $23 \mathrm{C} 1-2 ; 23 \mathrm{E} 1-2$ & +++ \\
\hline$D f(2 L) d r m-P 2$ & 23F3-4;24A1-2 & +++ \\
\hline$D f(2 L) e d 1$ & 24A2;24D4 & +++ \\
\hline$D f(2 L) B S C 109$ & $25 C 4 ; 25 C 8$ & ++++ \\
\hline$D f(2 L) E 110$ & 25F3-26A1;26D3-11 & ++++ \\
\hline$D f(2 L) B S C 6$ & 26D3-E1;26F4-7 & ++++ \\
\hline Df(2L)Dwee1-W05 & $27 C 2-3 ; 27 C 4-5$ & +++ \\
\hline Df(2L)XE-3801 & 27E2;28D1 & +++ \\
\hline$D f(2 L) B S C 142$ & $28 \mathrm{C} 3 ; 28 \mathrm{D} 3$ & ++++ \\
\hline$D f(2 L) B S C 143$ & 31B1;31D9 & +++ \\
\hline$D f(2 L) B S C 32$ & $32 \mathrm{~A} 1-2 ; 32 \mathrm{C} 5-\mathrm{D} 1$ & +++++ \\
\hline $\operatorname{Df}(2 L) B S C 147$ & $34 \mathrm{C} 1 ; 34 \mathrm{C} 6$ & ++++ \\
\hline Df(2L)Exel6049 & 40A5;40D3 & +++ \\
\hline$D f(2 R) w 45-30 n$ & 45A6-7;45E2-3 & ++++ \\
\hline$D f(2 R) C B 21$ & $48 \mathrm{E} ; 49 \mathrm{~A}$ & +++ \\
\hline Df(2R)Exel7130 & 50D4;50E4 & ++++ \\
\hline$D f(2 R)$ Exel7131 & 50E4;50F6 & +++++ \\
\hline$D f(2 R) B S C 11$ & 50E6-F1;51E2-4 & +++ \\
\hline$D f(2 R) B S C 550$ & $53 C 1 ; 53 C 6$ & ++++ \\
\hline$D f(2 R)$ robl-c & 54B17-C4;54C1-4 & +++ \\
\hline$D f(2 R) k 10408$ & $54 C 1-4 ; 54 C 1-4$ & +++ \\
\hline$D f(2 R) P 34$ & $55 \mathrm{E} 2-4 ; 56 C 1-11$ & ++++ \\
\hline Df(2R)Exel7162 & $56 \mathrm{~F} 11 ; 56 \mathrm{~F} 16$ & +++ \\
\hline$D f(2 R)$ or-BR6 & 59D5-10;60B3-8 & +++ \\
\hline$D f(3 L) M 21$ & $62 F ; 63 D$ & +++ \\
\hline$D f(3 L) G N 34$ & 63E6-9;64A8-9 & +++ \\
\hline$D f(3 L) X D / 98$ & $65 \mathrm{~A} 2 ; 65 \mathrm{E} 1$ & +++ \\
\hline$D f(3 L) B S C 33$ & 65E10-F1;65F2-6 & +++ \\
\hline$D f(3 L) 66 C-G 28$ & $66 B 8-9 ; 66 C 9-10$ & +++ \\
\hline$D f(3 L) B S C 13$ & $66 \mathrm{~B} 12-\mathrm{C} 1 ; 66 \mathrm{D} 2-4$ & +++ \\
\hline$D f(3 L) S c f-R 6$ & $66 \mathrm{E} 1-6 ; 66 \mathrm{~F} 1-6$ & +++++ \\
\hline$D f(3 L) B S C 10$ & 69D4-5;69F5-7 & +++ \\
\hline$D f(3 L) s t-f 13$ & 72C1-D1;73A3-4 & ++++ \\
\hline$D f(3 L) 81 k 19$ & $73 \mathrm{~A} 3 ; 74 \mathrm{~F}$ & +++++ \\
\hline$D f(3 L) k t o 2$ & 76B1-2;76D5 & +++ \\
\hline$D f(3 L) r i-79 C$ & 77B-C;77F-78A & ++++ \\
\hline$D f(3 L) r i-X T 1$ & 77E2-4;78A2-4 & ++++ \\
\hline Df(3L)ME107 & $77 F 3 ; 78 C 8-9$ & +++ \\
\hline$D f(3 L) B S C 249$ & 79B2;79D2 & ++++ \\
\hline$D f(3 R) p-X T 103$ & $85 \mathrm{~A} 2 ; 85 \mathrm{C} 1-2$ & +++++ \\
\hline$D f(3 R) M-K \times 1$ & $86 C 1 ; 87 \mathrm{~B} 1-5$ & ++++ \\
\hline Df(3R)ea & 88E7-13;89A1 & +++ \\
\hline$D f(3 R) s b d 104$ & $89 \mathrm{~B} 5 ; 89 \mathrm{C} 2-7$ & +++ \\
\hline$D f(3 R) P 115$ & $89 B 7-8 ; 89 E 7$ & ++++ \\
\hline$D f(3 R) 23 D 1$ & 94A3-4;94D1-4 & +++ \\
\hline$D f(3 R) c r b-F 89-4$ & 95D7-D11;95F15 & +++ \\
\hline Df(3R)Exel6197 & 95D8;95E5 & +++ \\
\hline$D f(3 R)$ Exel6202 & 96C9;96E2 & +++ \\
\hline$D f(3 R)$ Exel6203 & 96E2;96E6 & ++++ \\
\hline$D f(3 R) T I-P$ & $97 \mathrm{~A} ; 98 \mathrm{~A} 1-2$ & +++ \\
\hline$D f(3 R) / R 16$ & 97F1-2;98A & +++++ \\
\hline
\end{tabular}

${ }^{a}$ Each ' + ' represents 1.0 SD from the mean (i.e. 64.5\%) observed for Pink1 RNAi alone flies. Deficiencies that also suppressed the park RNAi wing posture phenotype (Table 2 ) are indicated in bold. 
Table 6 List of deficiencies showing lethal interactions with Pink1 knockdown

\begin{tabular}{|c|c|}
\hline Deficiencies & Breakpoints \\
\hline$D f(2 L) B S C 37$ & 22D2-3;22F1-2 \\
\hline$D f(2 L) d p p[d 14]$ & $22 \mathrm{E} 4-\mathrm{F} 2 ; 22 \mathrm{~F} 3-23 \mathrm{~A} 1$ \\
\hline$D f(2 L) C 144$ & $22 F 4-23 A 1 ; 23 C 2-4$ \\
\hline$D f(2 L) s c 19-8$ & $24 C 2-8 ; 25 C 8-9$ \\
\hline Df(2L)Exel6011 & 25C8;25D5 \\
\hline$D f(2 L) b 87 e 25$ & 34B12-C1;35B10-C1 \\
\hline$D f(2 L) T W 137$ & 36C2-4;37B9-C1 \\
\hline $\ln (2 R) b w[V D e 2 L] C y[R]$ & h42-h43;42A2-3 \\
\hline$D f(2 R) M 41 A 4$ & $41 \mathrm{~A} ; 41 \mathrm{~A}$ \\
\hline$D f(2 R) X 1$ & $46 C ; 47 A 1$ \\
\hline$D f(2 R) C X 1$ & $49 C 1-4 ; 50 C 23-D 2$ \\
\hline$D f(2 R) B S C 49$ & 53D9-E1;54B5-10 \\
\hline$D f(2 R) E D 4065$ & $60 C 8 ; 60 \mathrm{E} 8$ \\
\hline$D f(2 R) K r 10$ & $60 \mathrm{~F} 1 ; 60 \mathrm{~F} 5$ \\
\hline$D f(3 L) H R 119$ & $63 C 2 ; 63 F 7$ \\
\hline$D f(3 L) \operatorname{vin} 5$ & $68 A 2-3 ; 69 A 1-3$ \\
\hline$D f(3 L) \operatorname{vin} 7$ & $68 C 8-11 ; 69 B 4-5$ \\
\hline$D f(3 L) W 10$ & $75 A 6-7 ; 75 C 1-2$ \\
\hline$D f(3 L) E D 4978$ & 78D5;79A2 \\
\hline$D f(3 L) B S C 223$ & 79A3;79B3 \\
\hline Df(3R)Exel6144 & $83 A 6 ; 83 B 6$ \\
\hline$D f(3 R) p 712$ & 84D4-6;85B6 \\
\hline$D f(3 R) T-32$ & $86 \mathrm{E} 2-4 ; 87 \mathrm{C} 6-7$ \\
\hline$D f(3 R) D G 2$ & 89E1-F4;91B1-B2 \\
\hline$D f(3 R) D I-B X 12$ & 91F1-2;92D3-6 \\
\hline$D f(3 R) B 81$ & 99D3;3Rt \\
\hline
\end{tabular}

Above deficiencies, when crossed into the Pink1 knockdown background, significantly reduced the viability of Pink1 knockdown flies (less than five flies eclosed). Deficiencies that display a similar lethal interaction with park knockdown (Table 3 ) are indicated in bold.

reduced by $50 \%$ in dosage, also enhanced the wing phenotype in Pink1 null mutants (Table 7). Among 17 suppressor-containing cytological regions examined, 10 cytological regions, when reduced by $50 \%$ in dosage, also suppressed the wing phenotype in Pink1 null mutants (Table 7). Among 19 examined cytological regions that showed lethal interactions with both Pink1 and park in RNAi-based screens, 5 cytological regions, when reduced by $50 \%$ in dosage, also displayed the lethal phenotype in Pink1 null mutants (Table 8).

\section{Molecular characterization of the PD-interacting cytological region 21A1-21B7}

The PD-interacting cytological regions identified from above screens are relative large and contain a number of genes. As a first step towards molecular characterization of these PD-interacting cytological regions, we performed fine mapping in four selected PD-interacting cytological regions to identify corresponding PD-interacting genes. Those cytological regions were selected
Table 7 Analysis of the interaction between a Pink1 null mutation and cytological regions that modified both park-RNAi and pink1-RNAi wing phenotype

\begin{tabular}{|c|c|c|c|c|}
\hline \multirow[b]{2}{*}{ Deficiencies } & \multirow[b]{2}{*}{ Breakpoints } & \multicolumn{3}{|c|}{ Effects of modification } \\
\hline & & Pink1-RNAi & park-RNAi & Pink $1^{B 9}$ \\
\hline \multicolumn{5}{|l|}{ Enhancers } \\
\hline$D f(2 L) n e t-P M F$ & 21A1;21B7-8 & ++ & ++ & $n / d$ \\
\hline$D f(2 L) B S C 17$ & $30 \mathrm{C} 3-5 ; 30 \mathrm{~F} 1$ & ++ & ++ & $n / d$ \\
\hline$D f(2 L) B S C 50$ & 30F5;31B1 & +++ & ++++++ & En \\
\hline Df(2R)nap9 & $42 \mathrm{~A} 1-2 ; 42 \mathrm{E} 6-\mathrm{F} 1$ & ++ & +++++ & En \\
\hline$D f(2 R) c n 9$ & $42 \mathrm{E} ; 44 \mathrm{C}$ & ++ & ++ & En \\
\hline$D f(2 R) B S C 39$ & 48C5-D1;48D5-E1 & ++ & ++++ & En \\
\hline$D f(3 R) B S C 47$ & 83B7-C1;83C6-D1 & ++ & ++ & En \\
\hline$D f(3 R) T p / 10$ & $83 \mathrm{C} 1-2 ; 84 \mathrm{~B} 1-2$ & ++ & ++ & No \\
\hline \multicolumn{5}{|l|}{ Suppressors } \\
\hline$\overline{D f(2 L) B S C 106}$ & $21 \mathrm{~B} 7 ; 21 \mathrm{C} 2$ & - & - - & Su \\
\hline$D f(2 L) d p-79 b$ & 22A2-3;22D5-E1 & - - - & - - & No \\
\hline Df(2L)ed1 & $24 \mathrm{~A} 2 ; 24 \mathrm{D} 4$ & - & - - & $n / d$ \\
\hline Df(2L)BSC109 & $25 C 4 ; 25 C 8$ & - - & - & Su \\
\hline$D f(2 L) E 110$ & 25F3-26A1;26D3-11 & - - & - & $n / d$ \\
\hline Df(2L)BSC142 & 28C3;28D3 & - & -— & Su \\
\hline Df(2L)BSC143 & 31B1;31D9 & - & - & No \\
\hline Df(2R)Exel7131 & 50E4;50F6 & - - - & - - & Su \\
\hline Df(2R)BSC550 & $53 \mathrm{C} 1 ; 53 \mathrm{C} 6$ & -— & - & No \\
\hline Df(2R)robl-c & $54 \mathrm{~B} 17-\mathrm{C} 4 ; 54 \mathrm{C} 1-4$ & - & - & $n / d$ \\
\hline$D f(2 R) P 34$ & 55E2-4;56C1-11 & - - & - - & Su \\
\hline$D f(3 L) X D / 98$ & 65A2;65E1 & - & - & $n / d$ \\
\hline$D f(3 L) B S C 33$ & 65E10-F1;65F2-6 & - & - & $n / d$ \\
\hline Df(3L)66C-G28 & 66B8-9;66C9-10 & - & - & No \\
\hline$D f(3 L) S C f-R 6$ & 66Е1-6;66F1-6 & - - & - & Su \\
\hline$D f(3 L) B S C 10$ & 69D4-5;69F5-7 & - & -— & Su \\
\hline Df(3L)ME107 & 77F3;78C8-9 & - & - & No \\
\hline$D f(3 R) p-X T 103$ & $85 \mathrm{~A} 2 ; 85 \mathrm{C} 1-2$ & - - - & - - & Su \\
\hline Df(3R)sbd104 & $89 B 5 ; 89 C 2-7$ & - & - & $n / d$ \\
\hline$D f(3 R) P 115$ & 89B7-8;89E7 & - & - & Su \\
\hline$D f(3 R) c r b-F 89-4$ & 95D7-D11;95F15 & - & - & No \\
\hline Df(3R)Exel6202 & 96C9;96E2 & - & - & No \\
\hline Df(3R)Exel6203 & 96E2;96E6 & - - & - & Su \\
\hline
\end{tabular}

Abbreviations: $\mathrm{n} / \mathrm{d}$, not determined; Su, suppression; En, enhancement; No, no modification.

since they displayed strongest interactions with both park and Pink1.

From above screens, we found that reducing the dosage of the cytological region 21A1-21B7-8, deleted in the deficiency chromosome $D f(2 L)$ net-PMF, enhanced both park and Pink1 wing phenotype (Table 1 and 4). To identify the corresponding PD-interacting gene within this cytological region, we tested additional deficiency lines that carry smaller deletions within this region. We found that similar enhancement was observed when a smaller deficiency chromosome $D f(2 L)$ 
Table 8 Analysis of the interaction between a Pink1 null allele and deficiencies that exhibited lethal interactions with both park and Pink1 knockdown

\begin{tabular}{|c|c|c|}
\hline Deficiencies & Breakpoints & $\begin{array}{l}\text { Synthetic lethal } \\
\text { with pink } 1^{\mathrm{B9}}\end{array}$ \\
\hline$D f(2 L) B S C 37$ & $22 \mathrm{D} 2-3 ;$ 22F1-2 & No \\
\hline$D f(2 L) d p p[d 14]$ & 22E4-F2; 22F3-23A1 & Yes \\
\hline$D f(2 L) C 144$ & $22 \mathrm{~F} 4-23 \mathrm{~A} 1 ; 23 \mathrm{C} 2-4$ & Partial \\
\hline Df(2L)Exel6011 & $25 \mathrm{C} 8 ; 25 \mathrm{D} 5$ & No \\
\hline Df(2L)TW137 & $36 \mathrm{C} 2-4 ; 37 \mathrm{B9}-\mathrm{C} 1$ & No \\
\hline $\ln (2 R) b w[V D e 2 L] C y[R]$ & h42-h43;42A2-3 & Yes \\
\hline$D f(2 R) M 41 A 4$ & $h 44 ; 42 A 2$ & Yes \\
\hline$D f(2 R) \times 1$ & $46 C ; 47 A 1$ & $\mathrm{n} / \mathrm{d}$ \\
\hline$D f(2 R) C \times 1$ & 49C1-4;50C23-D2 & Yes \\
\hline$D f(2 R) B S C 49$ & 53D9-E1;54B5-10 & No \\
\hline$D f(2 R) E D 4065$ & $60 \mathrm{C} 8 ; 60 \mathrm{E} 8$ & \\
\hline$D f(2 R) K r 10$ & $60 F 1 ; 60 F 5$ & No \\
\hline Df(3L)HR119 & $63 C 2 ; 63 F 7$ & No \\
\hline Df(3L)vin5 & $68 A 2-3 ; 69 A 1-3$ & No \\
\hline$D f(3 L) \operatorname{vin} 7$ & 68C8-11;69B4-5 & No \\
\hline$D f(3 L) W 10$ & 75A6-7;75C1-2 & No \\
\hline Df(3L)ED4978 & 78D5;79A2 & No \\
\hline$D f(3 L) B S C 223$ & 79A3;79B3 & No \\
\hline Df(3R)Exel6144 & 83А6;83B6 & No \\
\hline$D f(3 R) p 712$ & 84D4-6;85B6 & $\mathrm{n} / \mathrm{d}$ \\
\hline$D f(3 R) T-32$ & $86 \mathrm{E} 2-4 ; 87 C 6-7$ & No \\
\hline$D f(3 R) D /-B \times 12$ & 91F1-2;92D3-6 & No \\
\hline
\end{tabular}

ED5878 was crossed into park or Pink1 knockdown background (Figure 3). Twenty two genes are disrupted in this deficiency chromosome, including $d b r$, galectin, CG11374, net, CG11376, Sam-S, CG4822, Gs1, CG31976, CG3709, CG11377, CG13694, CG31975, CG11455, Nhe1, CG3164, CG31974, CG3436, CG11454, CG33635, CG42399 and spen. Interestingly, we found that another smaller deficiency $D f(2 L)$ ED2809 in which

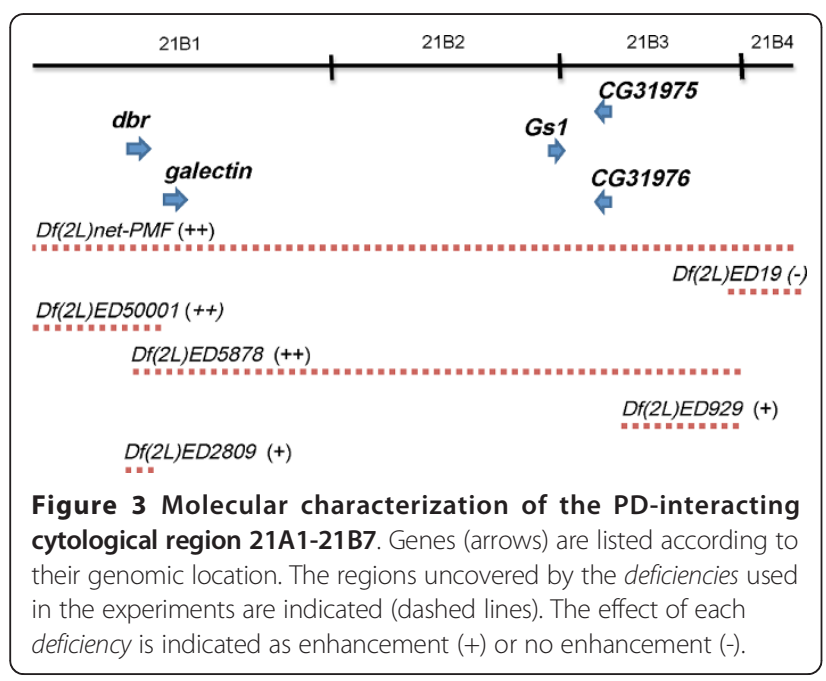

only the debra $(\mathrm{dbr})$ gene is deleted, also enhanced the park knockdown phenotype ( $50 \%$ increase in penetrance compared to park RNAi alone, $\mathrm{n}=104)$. Taken together, these results suggest strongly that $d b r$ is largely, if not entirely, responsible for the observed interaction with PD genes.

\section{Molecular characterization of two PD suppressor- containing cytological regions 21B7-21C2 and 50E4-50F6} Reducing the dosage of the cytological region 21B7-21C2, uncovered by the deficiency chromosome $D f(2 L) B S C 106$, suppressed both park and Pink1 wing phenotype (Table 2 and 5). From a collection of smaller deficiencies mapped within this region, we identified two overlapping deficiencies $D f(2 L) B S C 454$ and $D f(2 L) P i 3 K 21 B$, which like $D f(2 L) B S C 106$, both suppressed park and Pink1 wing phenotype (Figure 4A). The cytological region deleted in both $D f(2 L) B S C 454$ and $D f(2 L) P i 3 K 21 B$, contains four genes Hop, Pi3K21B, Plc21C and U2af38.

To further narrow down the PD-interacting gene within this region, we tested if any of above four genes interacts with PD genes. Among them, we found that knockdown the expression of Pi3K $21 B$ also significantly suppressed the Pink1 wing phenotype ( $48 \%$ decrease in penetrance compared to Pink1 RNAi alone, $\mathrm{n}=42$ ). This result suggests that $P i 3 K 21 B$ is the corresponding PD-interacting gene.

Reducing the dosage of the cytological region 50E4$50 \mathrm{~F} 6$, uncovered by the deficiency chromosome $D f(2 R)$ Exel7131, also suppressed both park and Pink1 knockdown wing phenotype (Table 2 and 5). However, another deficiency $D f(2 R) B S C 700$, in which the deleted cytological region partially overlaps with that affected in $D f(2 R)$ Exel7131, did not interact with park or Pink1. The cytological region deleted in $D f(2 R)$ Exel7131, but not in $D f(2 R) B S C 700$, carry nine genes (i.e. opa1-like, CG8485, CG8494, CG8503, Mdr50, Hsc70-5, CG8531, B4GalNAcTA and CG8547) (Figure 4B).

To test if the above genes interact with park or Pink1, we crossed available mutations into park or Pink1 knockdown background. We found that opa1 and $\beta 4$ GalNAcTA interact genetically with PD genes (Figure 4B). A heterozygous mutation of opa1 (i.e. opa $1^{\text {EY09863 }}$ ) significantly suppressed the park wing phenotype ( 95\% reduction in penetrance compared to park knockdown alone, $\mathrm{n}=83$ ). And heterozygous mutations of $\beta 4 \mathrm{Gal}$ NAcTA, Df (2R)ß4GalNAcTA ${ }^{[20.1]}$ (deleting both $\beta 4$ Gal$N A c T A$ and its neighboring gene CG8547) and $\beta 4 G_{\text {alNAcTA }}^{4.1}$ (deleting part of the $\beta 4$ GalNAcTA gene only), significantly suppressed the Pink1 wing phenotype (for $D f(2 R) \beta 4$ GalNAcTA ${ }^{[20.1]}$, $\sim 92 \%$ reduction in penetrance compared to Pink1 knockdown alone, $\mathrm{n}=62$; for $\beta 4$ GalNAcTA $^{4.1}, \sim 82 \%$ reduction in penetrance compared to Pink1 knockdown alone, $\mathrm{n}=59$ ). 


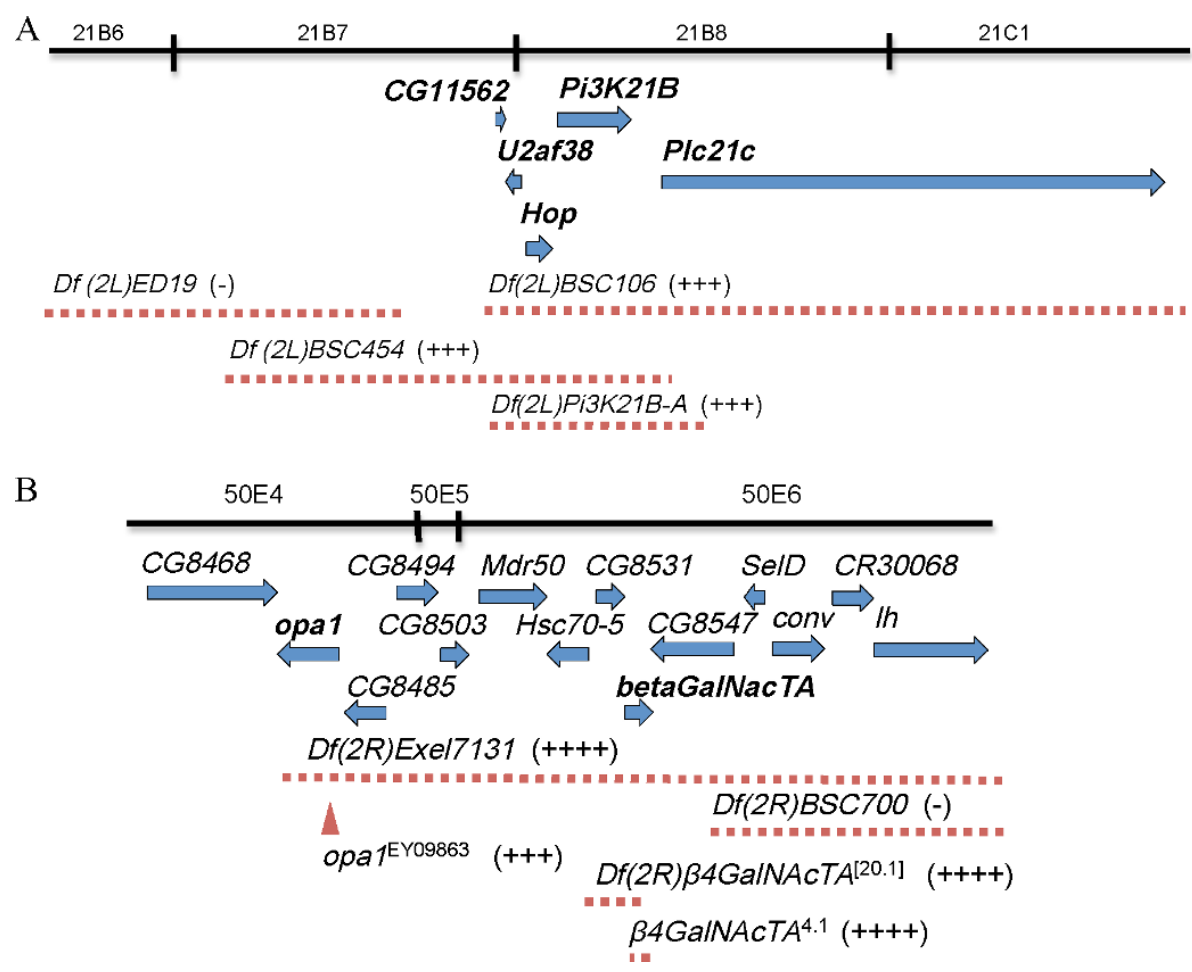

Figure 4 Molecular characterization of two PD suppressor-containing cytological regions 21B7-21C2 and 50E4-50F6. A, Characterization of the PD-interacting cytological region 21B7-21C2. B, Characterization of the PD-interacting cytological region 50E4-50F6. The regions uncovered by the deficiencies used in the experiments are indicated (dashed line). The effect of each deficiency is indicated as suppression (+) or no suppression (-). The genes (arrows) are listed according to their genomic location. opa ${ }^{\text {EY09863 }}$ is an opal loss-of-function allele in which a Pelement-containing sequence is inserted into the opal locus. Df(2R) $\beta 4 G$ GaINAcTA ${ }^{[20.1]}$ and $\beta 4 G$ GINAcTA $A^{4.1}$ were generated by imprecise excision [47]. In the Df(2R) $\beta 4$ GalNAcTA ${ }^{[20.1]}$ allele, both $\beta 4$ GalNAcTA and CG8531 are deleted, while 610 base-pair sequence in the $\beta 4$ GalNAcTA gene is deleted in the $\beta 4$ GaINACTA ${ }^{4.1}$ allele.

drp1 is the corresponding gene of the cytological region 22F4-23A3 that displayed lethal interaction with PD

genes

Two deficiencies, $D f(2 L) d p p^{[\mathrm{d} 14]}$ (22E4-F2;22F3-23A1) and $D f(2 L) C 144$ (22F4-23A1;23C2-4), caused lethality when heterozygous in park RNAi, Pink1 RNAi or Pink1 null mutant background (Table 8). A smaller deficiency (i.e. $D f(2 L) E D 136)$, which deletes the overlapping region defined by the above deficiencies, also caused partial lethality in the Pink1 null background (i.e. $\sim 50 \%$ reduction in viability compared to controls). The cytological region deleted in $D f(2 L) E D 136$ contains 29 genes, of which mutations in $d r p 1$ have been previously implicated as an enhancer of park and Pink1 mutant phenotypes $[16,33,34]$. Hence, we used a mutant allele for $d r p 1$ (i.e. $d r p 1^{\mathrm{KG03815}}$ ) to examine the potential interaction. Consistent with previous reports, we found that drp1 heterozygosity substantially enhanced the lethal phenotype in the Pink1 null background (i.e. $~ 82.8 \%$ reduction in viability compared to controls). This result strongly suggests that $d r p 1$ is the corresponding gene within the cytological region 22F4-23A3 that displayed lethal interaction with PD genes.

\section{Discussion}

In this study, we performed a genome-wide screen to isolate modifiers of PD genes. From this screen, we identified a number of cytological regions that interact with park and/or Pink1. Fine mapping of selected PDinteracting cytological regions led to the identification of corresponding PD-interacting genes. Among them, opa1 and $\operatorname{drp} 1$ have previously been implicated in Pink1/ park-mediated mitochondrial quality control pathways. In addition, we also identified debra, Pi3K21B, and $\beta 4$ GalNAcTA as novel PD-interacting genes.

While several previous studies suggest that park and Pink1 function in a common pathway to regulate mitochondrial function, cytological regions identified from our park- and Pink1-modifying screens do not completely overlap. For instance, among cytological regions showing lethal interactions with Pink1, about 81\% displayed similar interactions with park (Table 6). Among 
cytological regions modifying Pink1 wing phenotype, only $\sim 44 \%$ showed similar interactions with park (Table 4 and 5). One possible explanation is that park and Pink1 knockdown genetic background have different sensitivity, which may account for the difference in their interactions with some cytological regions. Alternatively or additionally, the molecular network involving Park and Pink1 may be more complex than a simplified linear pathway.

A previous study by Pallanck and colleagues screened a collection of P-element insertions (covering less than $10 \%$ of the fly genome) that modify the partial lethality of park null mutants [30]. However, since their screen was conducted in homozygous park null mutant background, less than $10 \%$ of the fly genome was covered. To increase the coverage, we developed an $R N A i$-based strategy, which allowed us to perform a F1 screen that covered $>80 \%$ of the fly genome. Several PD-interacting genes identified by Pallanck and colleagues in their previous screen [30], are located within PD-interacting cytological regions identified from our screen. For instance, Glutathione S-transferase 1 (Gst1) and Thioredoxin-2 (Trx-2) are located in PD-interacting cytological regions uncovered by $D f(2 R) B S C 49$ (Table 6) and $D f(2 L)$ N22-14 (Table 3), respectively.

While our screen using deficiencies greatly increases the coverage of genomic regions, there are several limitations. For instance, since cytological regions deleted in deficiency chromosomes contain a large number of genes (average $\sim 50$ ), it is possible that a cytological region containing $\mathrm{PD}$-interacting genes may not be identified from our screen if both enhancers and suppressors are located within the same region. Similarly, this may also make it difficult to identify the corresponding genes, especially if the strong modifying effect is due to the presence of multiple weak modifiers within the same region. Additionally, since those deficiency chromosomes used in our screen may carry second-site mutations contributing to the observed interactions, it is necessary to characterize independent point mutations and/or deletions mapped within the same region.

Our screen isolated two known PD-interacting genes $d r p 1$ and Opa1. $d r p 1$ encodes a GTPase (i.e. the dynamin-related protein 1) that has been previously implicated in regulating mitochondrial fission [35], while opa1 (optic atrophy 1) encodes for another dynaminrelated GTPase that promotes mitochondrial fusion $[36,37]$. Consistent with previous reports $[16,33,34]$, we showed that $d r p 1$ heterozygosity induced lethality prior to the adult stage in park or Pink1 knockdown background. We also showed that opa1 heterozygosity significantly suppressed the park-RNAi-induced wing phenotype. Similarly, previous reports showed that heterozygous mutations of opa1 suppressed indirect flight muscle degeneration and mitochondrial morphological phenotypes in Pink1 and park mutants [33,34]. Together, these observations underscore the importance of PD-interacting genes in mitochondrial fission and fusion to facilitate mitochondrial quality control.

Among the three novel PD-interacting genes (i.e. debra, Pi3K21B, and $\beta 4$ GalNAcTA) isolated from our screen, debra (determiner of breaking down of $\mathrm{Ci} a \mathrm{cti}$ vator) ( $d b r$ ) heterozygosity led to strong enhancement of the park-RNAi-induced wing phenotype. $d b r$ encodes a novel zinc-binding protein of 1007 amino-acid residues [38]. Cell culture studies showed that Dbr forms a complex with Slimb, a component of the SCF (Skpl, Cdc53 and $\mathrm{F}$ box) ubiquitin ligase complex, to mediate the polyubiquitination of the transcription factor Cubitus interruptus $(\mathrm{Ci})$ and thus targets $\mathrm{Ci}$ into the lysosome for degradation [38]. This raises the interesting possibility that Dbr functions together with Park in the ubiquitin-proteasome pathway for the control of protein quality. Reducing the dosage of $d b r$ may thus increase the accumulation of toxic protein substrates, leading to the enhancement of the park phenotype. In this context, it is worth noting that a recent study showed that reducing the level of $d b r$ also enhanced Ataxin3-induced neurodegeneration in Drosophila, which also resulted from accumulation of pathogenic proteins [39]. Additionally, since Dbr is a zinc-binding protein, Dbr may also play a role in regulating the level of intracellular zinc. Zinc dyshomeostasis has been shown to cause abnormalities in autophagy that are associated with Alzheimer's disease, Parkinson's disease, and Huntington's disease [40]. Thus, it is possible that in addition to its interaction with Park in the ubiquitin-proteasome pathway, Dbr may interact with the PD pathway by regulating autophagy.

Another novel-PD-interacting gene Pi3K21B, identified in our screen as a suppressor of PD wing phenotype, encodes an $\mathrm{SH} 2$ domain-containing adaptor protein that binds to the Drosophila class IA Phosphoinisitide 3 Kinase (PI3K), Pi3K92E/Dp110 [41]. It has been shown that class IA PI3-kinases are activated by nutrient-responsive insulin signalling to regulate cell growth and proliferation [42]. Loss of Pi3K21B-binding sites completely abrogates the activation of $\mathrm{Dp} 110$ by the insulin receptor, which decreased cell growth leading to reduced body size [43]. One possible explanation for the observed interaction between Pi3K21B and PD genes is that reducing the level of $P i 3 K 21 B$ may decrease insulin signaling and metabolic activities. This may be achieved by reducing the level of the TOR (target of rapamycin) signaling pathway. TOR can be activated by the PI3K/Akt pathway to regulate cell growth and metabolism (for review, see [44]). Recent studies show that reducing TOR signaling rescued $P D$ 
phenotypes in Drosophila by decreasing S6 kinasemediated 5'-Cap-dependent translation [45], and increasing 4E-BP-promoted 5'-Cap-independent translation [46]. Similarly, we speculate that Pi3K21B heterozygosity promotes 5 -Cap-independent translation by reducing TOR signaling, thus increasing the production of pro-survival factors leading to the suppression of PD phenotypes.

Characterization of the suppressors of the Pink1$R N A i$-induced wing phenotype also identified $\beta 4 \mathrm{Gal}$ NAcTA as a novel PD-interacting gene. $\beta 4$ GalNAcTA encodes for a $\beta$ 1,4-N acetlygalactosaminyltransferase that mediates the $\mathrm{N}$-glycosylation of protein substrates [47]. Drosophila adult mutants of $\beta 4$ GalNAcTA display severe locomotion abnormalities such as a low climbing index and coordination defects [48]. Glycosylation may affect protein function by diverse mechanisms, such as promoting protein stability, enabling protein recognition, altering ligand affinity and inhibiting protein activity [49]. For instance, abnormal glycosylation of alphadystroglycan interferes with its function leading to congenital muscular dystrophy [50]. Glycosylation may also contribute to the misfolding and accumulation of several proteins implicated in neurodegenerative disorders. For instance, glycosylation of tau and amyloid precursor protein (APP) may promote the formation and accumulation of pathogenic advanced glycosylation end-products (AGEs) [51]. In addition, $\alpha$-synuclein, the primary component of Lewy bodies in Parkinson's disease, is also modified by glycosylation [9]. This modification is hypothesized to affect the clearance of $\alpha$-synuclein aggregates [9]. We speculate that glycosylation mediated by $\beta 4$ GalNAcTA affects the stability and/or activity of components in the PD pathways, which may contribute to the accumulation of toxic proteins, increased sensitivity to oxidative damage and mitochondrial dysfunction. Future studies will be needed to elucidate the exact action of $\beta 4$ GalNAcTA in the PD pathways.

\section{Conclusion}

Systematic genetic screens covering $\sim 80 \%$ of the entire genome were performed to identify modifiers of the PD phenotype in Drosophila. From the screen, we identified a number of cytological regions that interact with park and/or Pink1. Fine mapping in selected PD-interacting cytological regions was performed, which identified debra, Pi3K21B and $\beta 4$ GalNAcTA as novel PD-interacting genes. Future characterization of other PD-interacting cytological regions will likely lead to the identification of additional PD-interacting genes.

\section{Acknowledgements}

We thank people in the labs of Yong Rao and Don van Meyel for suggestions and comments; the Bloomington Stock Center, VDRC, D. van
Meyel, N. Haines, T. Fon and M. Guo for fly stocks. This work was supported by a Team grant from Neuroscience Canada, and an operating grant (MOP14688) awarded to Yong Rao from Canadian Institutes of Health Research.

\section{Author details}

'Department of Biology, McGill University Health Centre, 1650 Cedar Avenue, Montreal, Quebec H3G 1A4, Canada. ${ }^{2}$ McGill Centre for Research in Neuroscience, Department of Neurology and Neurosurgery, Department of Medicine, McGill University Health Centre, 1650 Cedar Avenue, Montreal, Quebec H3G 1A4, Canada.

\section{Authors' contributions}

CF conducted all experiments, and was involved in writing the manuscript. YR supervised and wrote the manuscript. All authors read and approve the manuscript.

\section{Competing interests}

The authors declare that they have no competing interests.

Received: 14 February 2011 Accepted: 19 April 2011

Published: 19 April 2011

\section{References}

1. Kitada T, Asakawa S, Hattori N, Matsumine H, Yamamura Y, Minoshima S, Yokochi M, Mizuno Y, Shimizu N: Mutations in the parkin gene cause autosomal recessive juvenile parkinsonism. Nature 1998, 392:605-608.

2. Valente EM, Abou-Sleiman PM, Caputo V, Muqit MM, Harvey K, Gispert S, Ali Z, Del Turco D, Bentivoglio AR, Healy DG, Albanese A, Nussbaum R, Gonzalez-Maldonado R, Deller T, Salvi S, Cortelli P, Gilks WP, Latchman DS, Harvey RJ, Dallapiccola B, Auburger G, Wood NW: Hereditary early-onset Parkinson's disease caused by mutations in PINK1. Science 2004, 304:1158-1160

3. Valente EM, Salvi S, lalongo T, Marongiu R, Elia AE, Caputo V, Romito L, Albanese A, Dallapiccola B, Bentivoglio AR: PINK1 mutations are associated with sporadic early-onset parkinsonism. Ann Neurol 2004, 56:336-341.

4. Bertoli-Avella AM, Giroud-Benitez JL, Akyol A, Barbosa E, Schaap O, van der Linde HC, Martignoni E, Lopiano L, Lamberti P, Fincati E, Antonini A, Stocchi F, Montagna P, Squitieri F, Marini P, Abbruzzese G, Fabbrini G, Marconi R, Dalla Libera A, Trianni G, Guidi M, De Gaetano A, Boff Maegawa G, De Leo A, Gallai V, de Rosa G, Vanacore N, Meco G, van Duijn CM, Oostra BA, et al: Novel parkin mutations detected in patients with early-onset Parkinson's disease. Mov Disord 2005, 20:424-431.

5. Lucking CB, Durr A, Bonifati V, Vaughan J, De Michele G, Gasser T, Harhangi BS, Meco G, Denefle P, Wood NW, Agid Y, Brice A: Association between early-onset Parkinson's disease and mutations in the parkin gene. N Engl J Med 2000, 342:1560-1567.

6. Imai Y, Soda M, Takahashi R: Parkin suppresses unfolded protein stressinduced cell death through its E3 ubiquitin-protein ligase activity. J Biol Chem 2000, 275:35661-35664

7. Zhang Y, Gao J, Chung KK, Huang H, Dawson VL, Dawson TM: Parkin functions as an E2-dependent ubiquitin-protein ligase and promotes the degradation of the synaptic vesicle-associated protein. CDCrel-1 Proc Natl Acad Sci USA 2000, 97:13354-13359.

8. Imai Y, Soda M, Inoue H, Hattori N, Mizuno Y, Takahashi R: An unfolded putative transmembrane polypeptide, which can lead to endoplasmic reticulum stress, is a substrate of Parkin. Cell 2001, 105:891-902.

9. Shimura H, Schlossmacher MG, Hattori N, Frosch MP, Trockenbacher A, Schneider R, Mizuno Y, Kosik KS, Selkoe DJ: Ubiquitination of a new form of alpha-synuclein by parkin from human brain: implications for Parkinson's disease. Science 2001, 293:263-269.

10. Chung KK, Zhang Y, Lim KL, Tanaka Y, Huang H, Gao J, Ross CA, Dawson VL, Dawson TM: Parkin ubiquitinates the alpha-synucleininteracting protein, synphilin-1: implications for Lewy-body formation in Parkinson disease. Nat Med 2001, 7:1144-1150.

11. Staropoli JF, McDermott C, Martinat C, Schulman B, Demireva E, Abeliovich A: Parkin is a component of an SCF-like ubiquitin ligase complex and protects postmitotic neurons from kainate excitotoxicity. Neuron 2003, 37:735-749.

12. Pridgeon JW, Olzmann JA, Chin LS, Li L: PINK1 protects against oxidative stress by phosphorylating mitochondrial chaperone TRAP1. PLOS Biol 2007, 5:e172. 
13. Plun-Favreau H, Klupsch K, Moisoi N, Gandhi S, Kjaer S, Frith D, Harvey K, Deas E, Harvey RJ, McDonald N, Wood NW, Martins LM, Downward J: The mitochondrial protease HtrA2 is regulated by Parkinson's diseaseassociated kinase PINK1. Nat Cell Biol 2007, 9:1243-1252.

14. Kim Y, Park J, Kim S, Song S, Kwon SK, Lee SH, Kitada T, Kim JM, Chung J: PINK1 controls mitochondrial localization of Parkin through direct phosphorylation. Biochem Biophys Res Commun 2008, 377:975-980.

15. Guo M: What have we learned from Drosophila models of Parkinson's disease? Prog Brain Res 2010, 184:3-16.

16. Poole AC, Thomas RE, Andrews LA, McBride HM, Whitworth AJ, Pallanck LJ: The PINK1/Parkin pathway regulates mitochondrial morphology. Proc Natl Acad Sci USA 2008, 105:1638-1643.

17. Yang $Y$, Gehrke S, Imai Y, Huang Z, Ouyang Y, Wang JW, Yang L, Beal MF, Vogel H, Lu B: Mitochondrial pathology and muscle and dopaminergic neuron degeneration caused by inactivation of Drosophila Pink1 is rescued by Parkin. Proc Natl Acad Sci USA 2006, 103:10793-10798,

18. Park J, Lee SB, Lee S, Kim Y, Song S, Kim S, Bae E, Kim J, Shong M, Kim JM, Chung J: Mitochondrial dysfunction in Drosophila PINK1 mutants is complemented by parkin. Nature 2006, 441:1157-1161.

19. Clark IE, Dodson MW, Jiang C, Cao JH, Huh JR, Seol JH, Yoo SJ, Hay BA, Guo M: Drosophila pink1 is required for mitochondrial function and interacts genetically with parkin. Nature 2006, 441:1162-1166.

20. Dagda RK, Chu CT: Mitochondrial quality control: insights on how Parkinson's disease related genes PINK1, parkin, and Omi/HtrA2 interact to maintain mitochondrial homeostasis. J Bioenerg Biomembr 2009, 41:473-479.

21. Exner N, Treske B, Paquet D, Holmstrom K, Schiesling C, Gispert S, CarballoCarbajal I, Berg D, Hoepken HH, Gasser T, Kruger R, Winklhofer KF, Vogel F, Reichert AS, Auburger G, Kahle PJ, Schmid B, Haass C: Loss-of-function of human PINK1 results in mitochondrial pathology and can be rescued by parkin. J Neurosci 2007, 27:12413-12418.

22. Vives-Bauza C, Zhou C, Huang Y, Cui M, de Vries RL, Kim J, May J, Tocilescu MA, Liu W, Ko HS, Magrane J, Moore DJ, Dawson VL, Grailhe R, Dawson TM, Li C, Tieu K, Przedborski S: PINK1-dependent recruitment of Parkin to mitochondria in mitophagy. Proc Natl Acad Sci USA 2010, 107:378-383.

23. Ziviani E, Tao RN, Whitworth AJ: Drosophila parkin requires PINK1 for mitochondrial translocation and ubiquitinates mitofusin. Proc Natl Acad Sci USA 2010, 107:5018-5023.

24. Geisler S, Holmstrom KM, Skujat D, Fiesel FC, Rothfuss OC, Kahle PJ, Springer W: PINK1/Parkin-mediated mitophagy is dependent on VDAC1 and p62/SQSTM1. Nat Cell Biol 2010, 12:119-131.

25. Narendra DP, Jin SM, Tanaka A, Suen DF, Gautier CA, Shen J, Cookson MR, Youle RJ: PINK1 is selectively stabilized on impaired mitochondria to activate Parkin. PLOS Biol 2010, 8:e1000298.

26. Challa M, Malladi S, Pellock BJ, Dresnek D, Varadarajan S, Yin YW, White K, Bratton SB: Drosophila Omi, a mitochondrial-localized IAP antagonist and proapoptotic serine protease. Embo J 2007, 26:3144-3156.

27. Liu Z, Wang X, Yu Y, Li X, Wang T, Jiang H, Ren Q, Jiao Y, Sawa A, Moran T, Ross CA, Montell C, Smith WW: A Drosophila model for LRRK2-linked parkinsonism. Proc Natl Acad Sci USA 2008, 105:2693-2698.

28. Venderova K, Kabbach G, Abdel-Messih E, Zhang Y, Parks RJ, Imai Y, Gehrke S, Ngsee J, Lavoie MJ, Slack RS, Rao Y, Zhang Z, Lu B, Haque ME, Park DS: Leucine-Rich Repeat Kinase 2 interacts with Parkin, DJ-1 and PINK-1 in a Drosophila melanogaster model of Parkinson's disease. Hum Mol Genet 2009, 18:4390-4404.

29. Greene JC, Whitworth AJ, Kuo I, Andrews LA, Feany MB, Pallanck LJ: Mitochondrial pathology and apoptotic muscle degeneration in Drosophila parkin mutants. Proc Natl Acad Sci USA 2003, 100:4078-4083.

30. Greene JC, Whitworth AJ, Andrews LA, Parker TJ, Pallanck LJ: Genetic and genomic studies of Drosophila parkin mutants implicate oxidative stress and innate immune responses in pathogenesis. Hum Mol Genet 2005, 14:799-811.

31. Lee $T$, Luo $L$ : Mosaic analysis with a repressible cell marker for studies of gene function in neuronal morphogenesis. Neuron 1999, 22:451-461.

32. Brand $\mathrm{AH}$, Perrimon N: Targeted gene expression as a means of altering cell fates and generating dominant phenotypes. Development 1993, 118:401-415.

33. Deng H, Dodson MW, Huang H, Guo M: The Parkinson's disease genes pink1 and parkin promote mitochondrial fission and/or inhibit fusion in Drosophila. Proc Natl Acad Sci USA 2008, 105:14503-14508.
34. Park J, Lee G, Chung J: The PINK1-Parkin pathway is involved in the regulation of mitochondrial remodeling process. Biochem Biophys Res Commun 2009, 378:518-523.

35. Aldridge AC, Benson LP, Siegenthaler MM, Whigham BT, Stowers RS, Hales KG: Roles for Drp1, a dynamin-related protein, and milton, a kinesin-associated protein, in mitochondrial segregation, unfurling and elongation during Drosophila spermatogenesis. Fly (Austin) 2007, 1:38-46.

36. Alexander C, Votruba M, Pesch UE, Thiselton DL, Mayer S, Moore A, Rodriguez M, Kellner U, Leo-Kottler B, Auburger G, Bhattacharya SS, Wissinger B: OPA1, encoding a dynamin-related GTPase, is mutated in autosomal dominant optic atrophy linked to chromosome 3q28. Nat Genet 2000, 26:211-215.

37. Yarosh W, Monserrate J, Tong JJ, Tse S, Le PK, Nguyen K, Brachmann CB, Wallace DC, Huang T: The molecular mechanisms of OPA1-mediated optic atrophy in Drosophila model and prospects for antioxidant treatment. PLoS Genet 2008, 4:e6.

38. Dai $P$, Akimaru $H$, Ishii S: A hedgehog-responsive region in the Drosophila wing disc is defined by debra-mediated ubiquitination and lysosomal degradation of Ci. Dev Cell 2003, 4:917-928.

39. Bilen J, Bonini NM: Genome-wide screen for modifiers of ataxin-3 neurodegeneration in Drosophila. PLOS Genet 2007, 3:1950-1964.

40. Lee SJ, Koh JY: Roles of zinc and metallothionein-3 in oxidative stressinduced lysosomal dysfunction, cell death, and autophagy in neurons and astrocytes. Mol Brain 2010, 3:30.

41. Weinkove D, Leevers SJ, MacDougall LK, Waterfield MD: p60 is an adaptor for the Drosophila phosphoinositide 3-kinase, Dp110. J Biol Chem 1997, 272:14606-14610.

42. Weinkove D, Neufeld TP, Twardzik T, Waterfield MD, Leevers SJ: Regulation of imaginal disc cell size, cell number and organ size by Drosophila class I(A) phosphoinositide 3-kinase and its adaptor. Curr Biol 1999, 9:1019-1029.

43. Oldham S, Stocker H, Laffargue M, Wittwer F, Wymann M, Hafen E: The Drosophila insulin/IGF receptor controls growth and size by modulating PtdlnsP(3) levels. Development 2002, 129:4103-4109.

44. Hietakangas V, Cohen SM: Regulation of tissue growth through nutrient sensing. Annu Rev Genet 2009, 43:389-410.

45. Liu S, Lu B: Reduction of protein translation and activation of autophagy protect against PINK1 pathogenesis in Drosophila melanogaster. PLOS Genet 2010, 6:e1001237.

46. Tain LS, Mortiboys H, Tao RN, Ziviani E, Bandmann O, Whitworth AJ: Rapamycin activation of 4E-BP prevents parkinsonian dopaminergic neuron loss. Nat Neurosci 2009, 12:1129-1135.

47. Haines N, Irvine KD: Functional analysis of Drosophila beta1,4-Nacetlygalactosaminyltransferases. Glycobiology 2005, 15:335-346.

48. Haines N, Stewart BA: Functional roles for beta1,4-Nacetlygalactosaminyltransferase-A in Drosophila larval neurons and muscles. Genetics 2007, 175:671-679.

49. Ohtsubo K, Marth JD: Glycosylation in cellular mechanisms of health and disease. Cell 2006, 126:855-867.

50. Longman C, Brockington M, Torelli S, Jimenez-Mallebrera C, Kennedy C, Khalil N, Feng L, Saran RK, Voit T, Merlini L, Sewry CA, Brown SC, Muntoni F: Mutations in the human LARGE gene cause MDC1D, a novel form of congenital muscular dystrophy with severe mental retardation and abnormal glycosylation of alpha-dystroglycan. Hum Mol Genet 2003, 12:2853-2861.

51. Dei R, Takeda A, Niwa H, Li M, Nakagomi $Y$, Watanabe M, Inagaki T, Washimi Y, Yasuda Y, Horie K, Miyata T, Sobue G: Lipid peroxidation and advanced glycation end products in the brain in normal aging and in Alzheimer's disease. Acta Neuropathol 2002, 104:113-122.

\section{doi:10.1186/1756-6606-4-17}

Cite this article as: Fernandes and Rao: Genome-wide screen for modifiers of Parkinson's disease genes in Drosophila. Molecular Brain 2011 4:17. 